Fisioter Bras 2022;23(1):1-17

doi: $\underline{10.33233 / \mathrm{fb} . v 23 i 1.4880}$

\title{
ARTIGO ORIGINAL \\ Percepção de saúde, mobilidade e humor de mulheres participantes de caminhada em imersão
}

Perception of health, mobility and mood of women participating in immersion walk

Maria Cláudia Gatto Cardia, M.Sc. ${ }^{*}$ Maria Helena Morgami de Almeida**, Eliane Araújo de Oliveira, D.Sc. ${ }^{* *}$, José Eduardo Pompeu, D.Sc. ${ }^{* *}$, Erika Christina Gouveia e Silva, D.Sc. ${ }^{* *}$, Fátima Aparecida Caromano**

*Departamento de Fisioterapia do Centro de Ciências da Saúde da Universidade Federal da Paraíba (UFPB), João Pessoa, PB, Departamento de Fonoaudiologia, Fisioterapia e Terapia Ocupacional da Faculdade de Medicina da Universidade de São Paulo (FMUSP), São Paulo, SP, **Departamento de Fonoaudiologia, Fisioterapia e Terapia Ocupacional da Faculdade de Medicina da Universidade de São Paulo (FMUSP), São Paulo, SP, ${ }^{* * *}$ Departamento de Fisioterapia do Centro de Ciências da Saúde da Universidade Federal da Paraíba (UFPB), João Pessoa, PB

Recebido em 11 de agosto de 2021; aceito em 17 de janeiro de 2022.

Correspondência: Fátima Caromano, Laboratório de Saúde e Comportamento, Dep. FOFITO, Rua Cipotânia 51 Cidade Universitária USP 056160-000 Butantã 056160-000 São Paulo SP

Maria Cláudia Gatto Cardia: gattocardia@gmail.com Maria Helena Morgami de Almeida: hmorgani@usp.br Eliane Araújo de Oliveira: elianeao@gmail.com José Eduardo Pompeu: j.e.pompeu@usp.br

Erika Christina Gouveia e Silva: erikacgouveia@gmail.com

Fátima Aparecida Caromano: fcaromano@uol.com.br

\section{Resumo}

Estudos têm demonstrado que exercícios realizados em meio aquático produzem benefícios diversos para mulheres. Esta pesquisa objetivou avaliar a percepção subjetiva de saúde, mobilidade e humor de mulheres quinquagenárias saudáveis e sedentárias em decorrência de participação em programa de caminhada em imersão. Participaram 59 mulheres entre 50 e 59 anos, distribuídas em grupos de 10 a 12 pessoas. 
A atividade foi desenvolvida em piscina aquecida 2 vezes por semana, por 30 minutos, durante 12 meses. O programa foi dividido em 4 etapas de 3 meses com 5 tempos de avaliação. Para a avaliação subjetiva sobre o estado de saúde, mobilidade e humor, utilizou-se uma escala numérica de 0 a 10 . Breve relato sobre os principais efeitos do programa também foi analisado em seu conteúdo. Observou-se melhora na percepção de saúde $(p<0,001)$, na mobilidade $(p<0,001)$ e no humor $(p=0,003)$. Na análise de conteúdo, 49,4\% dos relatos relacionaram-se com a melhora da saúde; $22,9 \%$ com a autoestima e satisfação e 19,3\% com a capacidade física. O estudo reforça a necessidade deste tipo de prática para a saúde integral da mulher de "meia idade" como medida preventiva do processo de envelhecimento saudável.

Palavras-chave: hidroterapia; assistência integral à saúde; humor; senescência.

\section{Abstract}

Studies have demonstrated that exercises performed in aquatic environment produce several benefits for women. This research aimed at evaluating the subjective perception on health status, mobility, and mood of healthy and sedentary 50-year-old women as a consequence of participating in an immersion walk program. 59 women aged 50 to 59 years old participated, distributed in groups of 10 to 12 people. The intervention was conducted in a heated pool, twice a week, for 30 minutes for 12 months. The program was divided into 4 phases of 3 months, with 5 evaluation times. For the subjective assessment of the health status, mobility and mood, a number scale from zero to ten was used. A brief report on the main effects of the program, was also analysed in its content. Improvement in health status perception $(p<0,001)$, mobility $(p<0,001)$ and $\operatorname{mood}(p=0,003)$ was noticed. In the content analysis, $49,4 \%$ of speeches were related to health improvement; $22,9 \%$ to self-esteem and satisfaction and $19,3 \%$ were related to physical capacity. The study reinforces the need for this type of practice for the integral health of middle-aged women as a preventive measure of the healthy aging process.

Keywords: hydrotherapy; comprehensive health care; affect; aging.

\section{Introdução}

A idade ainda que possa ser traduzida objetivamente como anos de vida, tem definido ciclos e trajetórias de vida, idades ou segmentos geracionais como "infância", "juventude", "meia idade" ou "terceira idade" e desse modo expressam um modo de ser e de estar no mundo [1].

Se num passado recente ter 50 anos significava o início da velhice, hoje com o avanço da expectativa de vida temos uma melhor relação com a senescência, que se 
caracteriza pelo processo de envelhecimento saudável [2], e que na mulher comumente inicia-se no período pós-menopausa. Assim, esse prolongamento da vida trouxe um distanciamento entre a juventude e a velhice demarcado como "meia idade" [1,2].

A promoção da saúde no envelhecimento deverá levar em consideração ainda a questão do gênero. A presença de grande número de mulheres em idade avançada é, em muitas sociedades, um fenômeno demográfico recente e a preparação para seu envelhecimento saudável deve ser mais do que uma busca pessoal, e sim, um desafio para gestores de políticas públicas, quer seja pela simples justificativa populacional, econômica ou pelo débito histórico de desigualdade de poder entre homens e mulheres que impacta fortemente nas condições de saúde da mulher [3,4].

Especificamente no que se refere à mulher de meia idade surgem diversas questões que suscitam atenção e cuidado, dentre elas destacamos o declínio do ciclo vital em que cessa a sua capacidade procriativa; o apelo ao desempenho do papel de cuidadora de pais idosos e de mediadora de relações intergeracionais. Somam-se a essas cobranças sociais, a expectativa de que a mulher se submeta a ditatura da beleza e da jovialidade. Esses fatores são muitas vezes vivenciados como fontes de estresse e conflito gerando adoecimento [1-3].

Outras questões relacionadas ao processo saúde-doença, tais como o estilo e condições de vida e do trabalho também podem influenciar negativamente na senescência, sendo o sedentarismo um dos principais fatores para o declínio da capacidade funcional [5]. No entanto, grande parte das mulheres quinquagenárias contemporâneas tem se preocupado com seu estado de saúde buscando recursos para o autocuidado, incluindo a prática de atividades físicas que lhes proporcionem bemestar [6]

Programas simples, com atividades preventivas atrativas, desenvolvidas em grupo são de baixo custo e podem ser estimuladas como complemento à fisioterapia reabilitadora convencional. Estes programas conduzidos por profissionais especializados, com indicadores de evolução pré-definidos e bem controlados podem apresentar boa adesão e promover melhoras funcionais. E neste sentido, a fisioterapia aquática surge como uma excelente opção.

Imprescindível para nossa sobrevivência, a água também é uma fonte de prazer podendo ser um meio de lazer, de atividade física e de reabilitação. A imersão na água como meio para relaxamento e cura não é privilégio do homem moderno, sendo praticada há muitos séculos e de diversas formas [7]. Em seus primórdios, a hidroterapia, esteve mais relacionada à balneoterapia, à crenoterapia e à termoterapia [8]. Mais recentemente, programas de cinesioterapia, fundamentados e dirigidos para 
populações específicas, em ambientes controlados, têm sido desenvolvidos com diferentes tipos de exercícios a exemplo das caminhadas e corridas em imersão [8-11].

Exercícios terapêuticos em imersão proporcionam efeitos fisiológicos benéficos que envolvem respostas cardíacas, respiratórias, renais e musculoesqueléticas [12], podendo atuar também na redução da dor, melhora de estados de humor, qualidade de vida, e na percepção da imagem corporal [13].

Estudos têm demonstrado benefícios dos exercícios terapêuticos em imersão para mulheres no período pós-menopausa [14] e em senescentes saudáveis [15], no entanto, poucos estudos avaliaram a percepção de mulheres sobre os efeitos deste tipo de intervenção. Acredita-se que o relato das pacientes em relação aos efeitos decorrentes de uma intervenção terapêutica seja uma fonte de informação relevante, desde que explorada de forma controlada e sistemática. Categorias de respostas podem indicar o uso de biomarcadores adequados para análise de evolução física e serem usados como feedback mensuráveis.

Neste sentido, esta pesquisa teve o objetivo de avaliar a percepção subjetiva de saúde, mobilidade e humor de mulheres quinquagenárias saudáveis em decorrência de participação em programa de caminhada em imersão.

\section{Métodos}

Trata-se de um estudo quase-experimental, com duas metodologias de análise, a primeira de caráter quantitativo, longitudinal e descritivo referiu-se ao desenvolvimento e avaliação do programa de caminhada em imersão em quatro etapas. A segunda, de caráter qualitativo e exploratório, referiu-se a obtenção e análise de conteúdo de relato de cada uma das participantes em relação ao programa realizado [16]. O projeto foi aprovado pelo Comitê de Ética em Pesquisa da Faculdade de Medicina da Universidade de São Paulo (CAAE:66719817.1.0000.0065). Todos os sujeitos foram instruídos sobre os procedimentos de avaliação e tratamento, e concordaram em participar de forma voluntária assinando o Termo de Consentimento Livre e Esclarecido, conforme a Resolução 466/12 do Conselho Nacional de Saúde e declaração de Helsinki.

\section{Participantes}

Para fins de inclusão no estudo foram admitidas mulheres entre 50 e 59 anos, saudáveis e sedentárias.

Para o atestado de saúde as participantes apresentaram parecer médico afirmando que estavam em condições clínicas para frequentar programa de exercícios 
físicos de baixa a moderada intensidade em imersão em água aquecida, constatando não serem portadoras de doenças cardiopulmonares, musculoesqueléticas, neurológicas ou dermatológicas que limitassem a prática de hidroterapia. As participantes também afirmaram não fazer uso de medicamentos, e declararam não ter praticado qualquer atividade física específica ou caminhadas regulares (mínimo 30 minutos, pelo menos duas vezes por semana) há cinco anos, caracterizando o estado de sedentarismo. Foram critérios de exclusão a falta ao treinamento sem reposição na mesma semana, intercorrências clínicas ou sociais que impedissem a continuidade no programa e a falta de interesse nas atividades físicas durante as aulas.

\section{Procedimentos}

Foram organizados grupos de 10 a 12 participantes, com intervenção realizada 2 vezes por semana, por 30 minutos. A piscina com dimensão de 12 por 8 metros e profundidade de 1.3 metros permitiu a imersão até o nível das axilas e a temperatura se manteve em uma média de $31^{\circ} \mathrm{C} \pm 1^{\circ} \mathrm{C}$. As quatro etapas e atividades do programa estão detalhadas no Quadro 1.

Quadro 1 - Descrição das etapas e atividades do programa de caminhada em imersão na água (ver PDF anexo)

Instrumentos e análise de dados

Foi utilizada uma escala numérica com 10 centímetros de comprimento e marcas a cada 1 centímetro para a avaliação subjetiva sobre o estado de saúde, mobilidade e humor, no qual o zero indicava a pior situação possível e o 10 a melhor situação possível para cada variável. Os resultados foram analisados mediante estatística descritiva, taxa de variação absoluta e percentual e pelo teste não paramétrico de Wilcoxon, devido a não normalidade das variáveis estudadas, utilizando o programa estatístico SPSS Statistical Package for the Social Sciences, v. 22.

Após a última sessão, cada uma das participantes foi incentivada a desenvolver um relato escrito sobre os efeitos que acreditava estar relacionado com a prática dos exercícios que realizou. A análise de conteúdo [16], dos relatos neste protocolo, cumpre o importante papel de fornecer elementos subjetivos que não poderiam ser identificados por meio de instrumentos estruturados ou semiestruturados. Os relatos foram organizados em torno do tema norteador "possíveis benefícios do programa de imersão" 
e todas as respostas foram identificadas e categorizadas com base na ocorrência e frequência.

\section{Resultados}

O estudo contou inicialmente com 136 mulheres e terminou com 59, apresentando uma adesão ao programa de $43 \%$ (Diagrama 1).

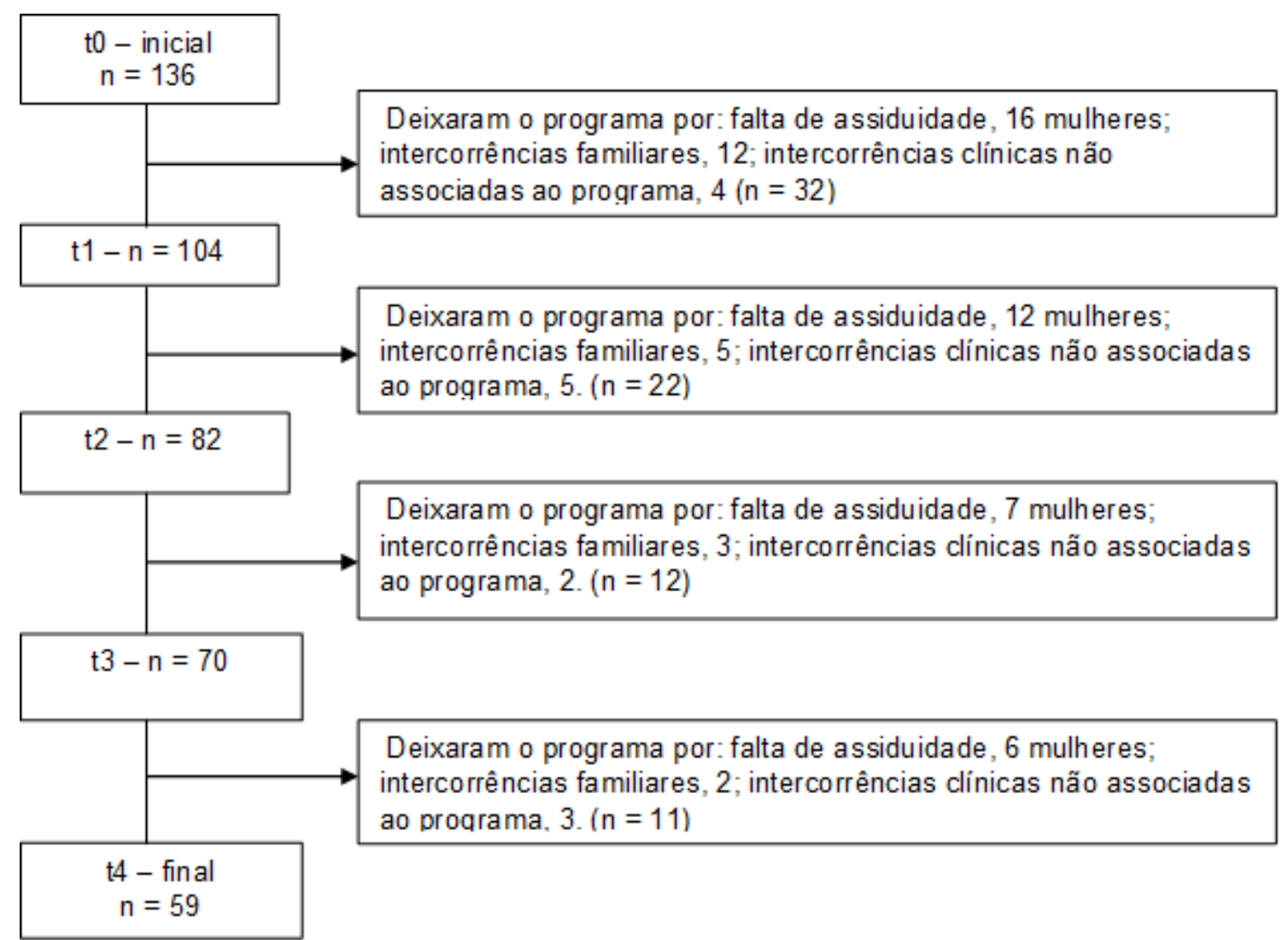

t0 = avaliação inicial; $\mathrm{t} 1$ = após o término da etapa $1 ; \mathrm{t} 2$ = após o término da etapa $2 ; \mathrm{t} 3$ = após o término da fase 3; 4 = após o término da fase $4 ; \mathrm{n}$ = número de pacientes; Fonte: Dados da pesquisa, 2018

Diagrama 1 - Distribuição da amostra

As intercorrências familiares corresponderam a: mudança de bairro, mudança de cidade, mudança na estrutura familiar, morte em família, nascimento de neto e desemprego em família e intercorrências clínicas devido a gripes, pneumonia, fratura, dermatite, anemia e dengue.

O grupo foi formado por mulheres quinquagenárias, com média de 52,9 $\pm 1,2$ anos; com escolaridade média de 10,9 $\pm 2,8$ anos; estatura mediana de 162,6 $\pm 3,2 \mathrm{~cm}$; e a maioria (52.5\%) apresentava sobrepeso. Eram profissionalmente ativas $78 \%$ e $49.2 \%$ eram casadas. 
Avaliação da percepção subjetiva do estado de saúde, da mobilidade e do humor das participantes pela escala numérica de 0 a 10

$\mathrm{Na}$ avaliação subjetiva, realizada com a escala numérica, houve melhora significativa nas 3 variáveis estudadas (Tabela I).

Tabela I - Análise quantitativa da percepção de saúde, mobilidade e humor em cada etapa do programa

\begin{tabular}{llllll}
\hline Variáveis & Mediana & $\begin{array}{l}\text { Mínimo e } \\
\text { máximo }\end{array}$ & $\begin{array}{l}\text { Wilcoxon P } \\
\text { (Sig) }\end{array}$ & $\begin{array}{l}\text { Variação } \\
\text { absoluta }\end{array}$ & $\begin{array}{c}\text { Percentual } \\
\text { (delta\%) }\end{array}$ \\
\hline Saúde_t0 & 7,0 & $5-10$ & 0,058 & 0,22 & 4,25 \\
Saúde_t1 & 7,0 & $5-10$ & 0,007 & 0,29 & 5,35 \\
Saúde_t2 & 7,0 & $6-10$ & 0,340 & 0,08 & 1,27 \\
Saúde_t3 & 7,0 & $5-10$ & $<0,001$ & 0,34 & 5,29 \\
Saúde_t4 & 8,0 & $6-10$ & $<0,001$ & 0,93 & $\mathbf{1 6 , 1 3}$ \\
Mobilidade_t0 & 6,0 & $5-9$ & $<0,001$ & 0,61 & 10,21 \\
Mobilidade_t1 & 7,0 & $6-9$ & 0,004 & 0,25 & 3,76 \\
Mobilidade_t2 & 7,0 & $6-10$ & $<0,001$ & 0,27 & 3,98 \\
Mobilidade_t3 & 8,0 & $6-10$ & 0,002 & 0,22 & 3,13 \\
Mobilidade_t4 & 8,0 & $6-10$ & $<0,001$ & $\mathbf{1 , 3 6}$ & $\mathbf{2 2 , 1 5}$ \\
Humor_t0 & 7,0 & $4-9$ & 0,051 & 0,20 & 4,51 \\
Humor_t1 & 7,0 & $5-9$ & 0,012 & 0,17 & 2,09 \\
Humor_t2 & 7,0 & $5-10$ & 0,317 & 0,07 & 1,27 \\
Humor_t3 & 7,0 & $6-10$ & 0,206 & 0,07 & 1,02 \\
Humor_t4 & 7,0 & $6-10$ & 0,002 & 0,51 & $\mathbf{9 , 6 9}$ \\
\hline
\end{tabular}

$\mathrm{p} \mathrm{();} \mathrm{t0} \mathrm{(Avaliação} \mathrm{inicial);} \mathrm{t1} \mathrm{(após} \mathrm{o} \mathrm{término} \mathrm{da} \mathrm{etapa} \mathrm{1);} \mathrm{t2} \mathrm{(após} \mathrm{o} \mathrm{término} \mathrm{da} \mathrm{etapa} \mathrm{2);} \mathrm{t3} \mathrm{(após} \mathrm{o} \mathrm{término}$ da fase 3); t4 (após o término da fase 4)

A percepção inicial do estado de saúde teve uma média regular de 6,95 \pm 1,4, mas foi considerada alta (entre boa, muito boa e excelente) por $25,4 \%$ das participantes, com nota 8 atribuída por $8,5 \%$ das mulheres; 9 por $10,2 \%$ e 10 (nota máxima) por $6,8 \%$ delas.

A mobilidade também teve uma média regular de 6,56 \pm 0,9 na avaliação inicial, sendo considerada alta por $18,6 \%$ das participantes, com nota 8 atribuída por $15,3 \%$ das mulheres e 9 por 3,4\%. A mobilidade foi considerada melhor desde a etapa 1 quando 52,6\% das participantes já atribuíram maior valor que na avaliação inicial e, na etapa final (t4), 86,4\% das mulheres consideraram ter melhor mobilidade, $10,2 \%$ relataram ter a mesma mobilidade que no início do programa e apenas 3,4\% tiveram a percepção de piora na mobilidade.

A percepção inicial do estado de humor teve igualmente uma média regular de $6,95 \pm 1,2$, sendo considerada alta por $40,7 \%$ das participantes, com nota 8 atribuída por $33,9 \%$ das mulheres e 9 por $6,8 \%$.

Apesar do alto número de participantes ter atribuído notas acima de 8 na avaliação inicial, conseguimos observar os efeitos positivos do programa na percepção da saúde com melhora de $16,13 \%$; da mobilidade com melhora de $22,15 \%$ e do estado 
de humor com de melhora de 9,69\% através da análise de variação percentual (t0-t4) (Gráfico 1).

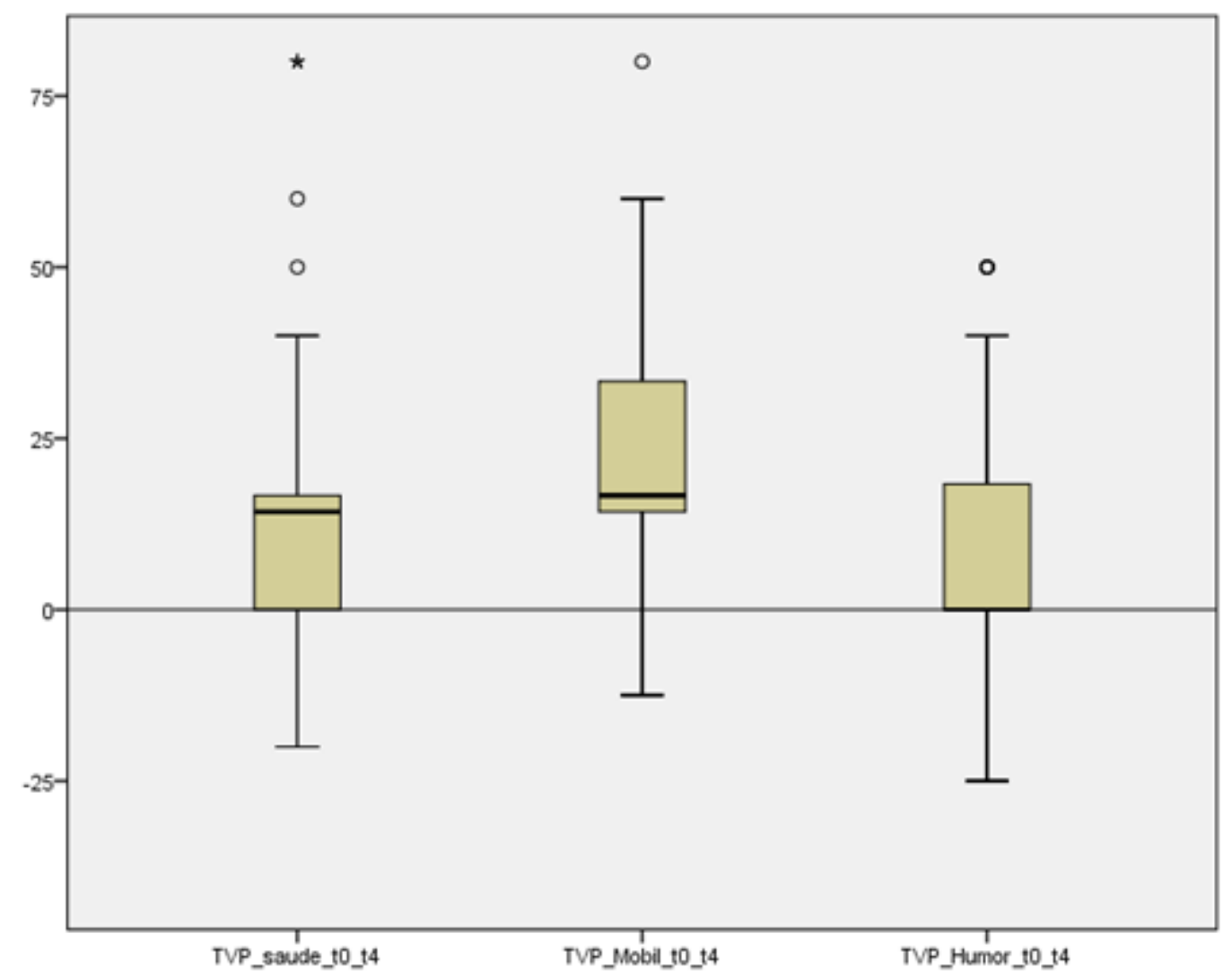

TVP_saúde = variável saúde; TVP_mobil $=$ variável mobilidade; TVP_humor $=$ variável humor; t0 = avaliação inicial; $\mathrm{t} 1$ = após o término da etapa $1 ; \mathrm{t} 2$ = após o término da etapa 2 ; $\mathrm{t} 3$ = após o término da fase 3; $\mathrm{t} 4$ = após o término da fase 4; $\mathrm{n}$ = número de pacientes; Fonte: Dados da pesquisa, 2018

Gráfico 1 - Distribuição da taxa de variação percentual do início e final do programa (to-t4) do estado de saúde, mobilidade e estado de humor

Análise de conteúdo do relato aberto das participantes no final do programa

Os relatos das participantes sobre o programa, realizados após a última sessão, foram compostos em seu conjunto por 83 relatos feitos por $94,9 \%$ das mulheres. A partir da análise de conteúdo destes relatos foram identificadas cinco categorias temáticas com 23 subcategorias, conforme descrito na tabela II. 
Tabela II - Análise qualitativa dos comentários realizados no final do programa

\begin{tabular}{|c|c|c|c|}
\hline Domínios & Categorias & $\mathrm{n}$ & Freq $\%$ \\
\hline $\begin{array}{l}\text { Mobilidade e } \\
\text { capacidade física }\end{array}$ & $\begin{array}{l}\text { andar melhor, com mais confiança ou maior resistência }-6 \\
\text { subir escadas (melhor, sem medo ou mais confiante) - } 3 \\
\text { me equilibro melhor }-1 \\
\text { mais forte }-5 \\
\text { mais ágil }-1\end{array}$ & 16 & $19,3 \%$ \\
\hline $\begin{array}{l}\text { Autoestima e } \\
\text { satisação } \\
\text { (sentir-se melhor, } \\
\text { mais Confiante e } \\
\text { mais feliz) }\end{array}$ & $\begin{array}{l}\text { mais sexy }-2 \\
\text { se sente melhor com o corpo ou } \\
\text { gosta mais do corpo agora }-5 \\
\text { se sente mais feliz - } 4 \\
\text { mais confiante, mais confiante no corpo e mais animada } \\
\text { com a vida, durmo e acordo mais animada }-5 \\
\text { adorei ter começado este programa }-1 \\
\text { mais coragem para enfrentar novos desafios }-1 \\
\text { mais solta }-1\end{array}$ & 19 & $22,9 \%$ \\
\hline Aspectos sociais & $\begin{array}{l}\text { tenho feito mais amigos - } 1 \\
\text { estou mais sociável }-1 \\
\text { percebo que as pessoas veem que estou mudando }-1\end{array}$ & 3 & $3,6 \%$ \\
\hline Estado de saúde & $\begin{array}{l}\text { corpo menos dolorido }-8 \\
\text { menos inchaço }-6 \\
\text { intestino funciona melhor }-5 \\
\text { qualidade do sono }-10 \\
\text { maior disposição }-11 \\
\text { mais tranquila }-1 \\
\end{array}$ & 41 & $49,4 \%$ \\
\hline Alimentação & $\begin{array}{l}\text { Sentia muita fome }-2 \\
\text { estimulada para controlar alimentação }-2\end{array}$ & 4 & $4,8 \%$ \\
\hline Total & & 83 & $100 \%$ \\
\hline
\end{tabular}

$\mathrm{n}$ = número de pacientes; Freq = frequência (\%); Fonte: Dados da pesquisa, 2018

Discussão

Este estudo mostrou que, por meio de informações objetivas foi possível descrever um quadro de percepções subjetivas referentes aos prováveis benefícios de programa anual de caminhada em imersão, na modalidade grupal. Foram conhecidas percepções dos participantes sobre a saúde, a mobilidade e o estado de humor em mulheres quinquagenárias saudáveis, a partir de indicações qualitativas quantificáveis através de uma escala numérica de zero a dez. Percepções adicionais foram expressas por meio de relatos escritos. Considera-se que, em conjunto, a análise dessas informações, revelou a percepção subjetiva destas mulheres sobre os efeitos do programa.

São poucos os estudos que utilizaram caminhada em imersão como recurso terapêutico e dentre eles apenas dois estudos [10,11] observaram os efeitos da caminhada na água nos aspectos psicossociais. O estudo de Katsura et al. [10] avaliou os efeitos de um treinamento de exercícios aquáticos utilizando um novo equipamento de resistência à água no desempenho do exercício e na promoção dos estados de humor (Profile Of Mood States - POMS) de 20 idosos saudáveis e concluíram que o treinamento melhorou a capacidade de caminhada, bem como a pontuação de tensão e ansiedade do POMS. 
Arrieiro [11] utilizou o autorrelato de dor, função física e rigidez articular, por meio da aplicação do Western Ontario and Mc Master Universities Osteoarthritis Index (WOMAC) e analisou a qualidade de vida, por meio do questionário Medical Outcomes Study 36-Item Short Form Health Survey (SF-36) tendo observado uma relação direta entre a melhora dos aspectos emocionais e a melhora do quadro clínico de indivíduos com osteoartrose de joelho.

Estudos de Sabioni, Galante \& Pelá [17] e Santana et al. [18], realizados com mulheres entre 31 e 80 anos e com terapia aquática, analisaram o conteúdo das falas dos participantes a respeito da intervenção, todavia a terapia aquática não foi especificamente a caminhada. Embora com método de pesquisas diferentes do utilizado no presente estudo, conteúdos semelhantes foram relevados.

Nossos resultados demonstraram um incremento positivo e significativo no estado de saúde entre as medidas t1-t2 ( $p=0,007)$, t3-t4 $(p<0,001)$ e t0-t4 $(p<0,001)$, com variação percentual do ganho entre a medida inicial e final de $16,13 \%(p<0,001)$. A maior parte dos relatos $(49,4 \%)$ foi relacionada com a melhora geral referida da saúde, como nos exemplos: "diminuição da dor, do inchaço, melhor funcionamento do intestino, melhora na qualidade do sono, mais disposição e tranquilidade".

Estado de saúde é uma condição subjetiva influenciada por fatores culturais, socioeconômicos e biológicos e reflete a conjuntura de uma época e lugar, e embora a epidemiologia expresse a saúde por meio de números, admitem-se indicadores subjetivos do estado de saúde que podem ser expressos através da narrativa na primeira pessoa, aquela que sente a dor e sofrimento ou felicidade e prazer [19].

Sabioni, Galante \& Pelá [17] relataram que a hidroterapia foi apresentada como algo importante na vida das mulheres estudadas, por proporcionar bem-estar, melhora na qualidade de vida e alívio da dor. Estes autores registraram $24,5 \%$ das falas relacionadas à sensação de bem-estar.

O estudo de Gomes \& Zazá [20], com 20 mulheres idosas (69,7 \pm 7,1 anos) demonstrou que, entre os motivos mais importantes para praticarem atividade física estavam melhorar ou manter o estado de saúde, prevenir doenças e aprender novas atividades.

Também foi observada uma melhora significativa na mobilidade das mulheres participantes em todos os momentos de avaliação: t0-t1 ( $p<0,001)$; t1-t2 ( $p=0,004)$; t2-t3 $(p<0,001)$ e t3-t4 ( $p=0,002)$ apresentando uma variação percentual do ganho entre a medida inicial (t0) e final (t4) de 22,15\% ( $p<0,001)$ na mobilidade percebida pelas participantes. Quanto às narrativas relacionadas à mobilidade e capacidade física, $19,3 \%$ das mulheres expressaram sentir-se "mais ágeis, com maior confiança para andar e subir escadas". 
Estes dados corroboram os estudos de Sabioni, Galante \& Pelá [17] que registraram falas relacionadas à mobilidade e à capacidade física; e de Santana [18] com relato específico de melhora de movimentos e sensação de relaxamento muscular. É comum a estes estudos e ao nosso, o reconhecimento do efeito do programa de intervenção nos aspectos físicos de origem musculoesquelética.

A mobilidade é um constructo importante para a percepção do estado de saúde e pode ser influenciada diretamente pela atividade física. Nessa perspectiva, a Sociedade Brasileira de Medicina do Esporte (SBME) identificou relação inversa entre o exercício praticado regularmente e as principais causas de morte na mulher pósmenopausa [21].

Sobre os estados de humor, o estudo de validação do instrumento de avaliação do perfil de estados de humor (POMS) apontou uma associação positiva entre prática de atividades físicas e desportivas e os estados emocionais e a saúde mental [22].

Os resultados deste estudo apontaram uma melhora significativa nas avaliações entre o t1-t2 ( $p=0,012)$ e t0-t4 ( $p=0,002)$ e uma análise de variação percentual (t0-t4) com melhora de 9,69\% ( $p=0,002)$ no estado de humor. Entretanto deve-se considerar que a avaliação inicial das participantes já apresentava bom escore inicial e apesar do "efeito teto" houve uma variação percentual de melhora ainda que mais baixa quando comparada às variáveis estado de saúde e mobilidade [23].

Sabioni, Galante \& Pelá [17] não apontam nenhuma fala específica com a palavra humor, no entanto o estado de humor pode ser percebido em diversas falas do referido estudo, em que também se evidencia o prazer em realizar atividades em imersão. E o estudo de Vieira, Rocha \& Porcu [24], realizado com mulheres com idade média de 43,33 \pm 3,56 anos e diagnóstico de depressão moderada, mostrou que um programa de hidroginástica de 12 semanas diminuiu a depressão e melhorou o humor das participantes do grupo experimental e não alterou o do grupo controle.

Da mesma forma, o estudo de Agra et al. [2], com mulheres com idade variando entre 55 e 75 anos, submetidas a um programa de 12 meses de exercícios aquáticos, alongamentos e fortalecimento muscular, também apontou para melhoras significativas na depressão, imagem corporal, autoestima e no estado de humor.

Queiroz Júnior et al. [6] ressaltam que benefícios psicossociais, como o alívio da depressão, o aumento da autoconfiança e a melhora da autoestima podem ser percebidos pelas pessoas que praticam regularmente exercícios físicos. Belza et al. [25], em um estudo com intervenção com exercícios aquáticos em adultos com osteoartrite, encontraram que a qualidade do bem-estar e o humor deprimido melhoraram para os aderentes, mas não para os não aderentes. 
No estudo de Gomes \& Zazá [19], o estado de humor não foi considerado especificamente, porém "aumentar o contato social" (85\%), e "a autoestima" (82,5\%) foram destacados como aspectos relevantes da intervenção pelos participantes.

Das 136 mulheres que iniciaram o programa de caminhada em imersão na água, 59 o finalizaram um ano após seu início, apresentando uma adesão de 43\%. Considerando que adesão possa significar concordância ativa, consciente e realmente voluntária, admite-se que as 59 assíduas participantes tenham extraído relevante proveito do programa, o que se expressa na melhora dos estados de saúde, humor e mobilidade e nos relatos positivos associados.

Por outro lado, a não-adesão é bastante comum em programas de atividade física, e foi observada neste estudo. Para aumentar a adesão, Belza et al. [25] reforçaram a necessidade de aprimoramento dos métodos com maior atenção ao papel de fatores intrínsecos, como autoeficácia e sistemas de crença.

Acreditamos que, neste estudo, a prática em grupo, com objetivos a serem atingidos pré-estabelecidos, níveis de dificuldade crescente das atividades, supervisão constante e especializada, associados ao conforto da prática de exercícios em imersão na água termoneutra influenciaram positivamente na adesão por parte das participantes.

\section{Conclusão}

O estudo mostrou os efeitos benéficos de um programa de caminhada em imersão na água com duração de um ano, no estado de saúde, na mobilidade e no estado de humor, de acordo com a percepção subjetiva de mulheres quinquagenárias. Desta forma, reforçamos a necessidade deste tipo de prática para a saúde integral da mulher de "meia idade" como medida preventiva do processo de envelhecimento saudável.

\section{Vinculação acadêmica}

Este artigo é parte da Tese "Efeitos da caminhada em imersão em mulheres quinquagenárias saudáveis" do programa de Doutorado em Ciências da Reabilitação da Faculdade de Medicina da Universidade de São Paulo (FMUSP)

\section{Conflitos de interesse}

Não há conflito de interesse

\section{Fonte de financiamento}

Não há fonte de financiamento

\section{Contribuição dos autores}

Concepção e desenho do estudo: Cardia MCG, Almeida MHM, Caromano FA; Coleta de dados: Cardia MCG, Almeida MHM, Caromano FA; Análise e interpretação dos dados: Cardia MCG, Almeida MHM, Caromano FA, Oliveira EA, Pompeu JE; Elaboração e revisão crítica do manuscrito: Cardia MCG, Almeida MHM, Caromano FA, Oliveira EA, Pompeu JE, Silva ECG; Aprovação da versão final do manuscrito: Cardia MCG, Almeida MHM, Caromano FA, Oliveira EA, Pompeu JE, Silva ECG 
1. Motta ABD. Mulheres entre o cuidado de velhos/as e a reprodução de jovens em famílias no Brasil. Ex Aequo [Internet]. 2012 [cited 2022 Jan 14];26:87-101. Available from: http://scielo.pt/pdf/aeq/n26/n26a08.pdf

2. Agra KOA, Borges AEA, Araújo KMB, Carvalho SMCR, Barreto JM, Oliveira EA. A terapia aquática como coadjuvante na variação do humor em mulheres pósmenopáusicas. Rev Bras Ciênc Saúde 2014;17(4):327-34. doi: 10.4034/RBCS.2013.17.04.02

3. Assis LTM, Fernandes BF. Saúde da mulher: a enfermagem nos programas e políticas públicas nacionais no período de 1984 a 2009. Revista Mineira de Enfermagem [Internet]. 2011[cited 2022 Jan 14];15(3):356-64. Available from: http://www.reme.org.br/artigo/detalhes/45

4. Vagetti GC, Boneti MN, Barbosa Filho VC, De Oliveira V, Ferreira Cancian C, Mazzardo $\mathrm{O}$, et al. Domínios da qualidade de vida associados à percepção de saúde: um estudo com idosas de um programa de atividade física em bairros de baixa renda de Curitiba, Paraná, Brasil. Ciênc Saúde Coletiva 2013;18:3483-93. doi: 10.1590/S141381232013001200005

5. Tairova OS, DeLorenzi DRS. Influência do exercício físico na qualidade de vida de mulheres na pós-menopausa: um estudo caso-controle. Rev Bras Geriatr Gerontol 2011;14(1):135-45. doi: 10.1590/S1809-98232011000100014

6. Queiroz Júnior CA, Castellano SM, De Paulo TRS, Moreira WW, Simões R. Motivos de adesão das mulheres idosas participantes dos programas públicos de exercícios físicos em Uberaba-MG. Colec Pesq Educ Fis [Internet]. 2012 [cited 2022 Jan 14];11(4):13542. Available from: https://fontouraeditora.com.br/periodico/upload/artigo/967_1503345505.pdf

7. Pinto LLT, Morbeck DI, Moreira RM. Análise reflexiva sobre os benefícios da hidroginástica para a saúde de pessoas idosas. Saúde e Pesquisa [Internet]. 2014 [cited 2022 Jan 14];7(2):353-59. Available from: https://periodicos.unicesumar.edu.br/index.php/saudpesq/article/view/3084/2385

8. Chevutschi A, Dengremont B, Lensel G, Pardessus V, Thevenon A. La balnéothérapie au sein de la littérature: Applications thérapeutiques. Kinesitherapie 2007;7(71):14-23.

9. Candeloro J, Caromano F. Efeito de um programa de hidroterapia na flexibilidade e na força muscular de idosas. Rev Bras Fisioter 2007;11:303-09. doi: 10.1590/S141335552007000400010

10. Katsura Y, Yoshikawa T, Ueda SY, Usui T, Sotobayashi D, Nakao H, et al. Effects of aquatic exercise training using water-resistance equipment in elderly. Eur $\mathrm{J}$ Appl Physiol 2010;108(5):957-64. doi: 10.1007/s00421-009-1306-0

11. Arrieiro AN. Efeitos do treinamento aeróbio por meio de caminhada na água ou no solo no desempenho físico-funcional e na qualidade de vida de mulheres idosas com osteoartrite de joelho [Dissertação] [Internet]. Diamantina: Universidade Federal dos 
Vales dos Jequitinhonha e Mucuri, 2011;115p. Available from:

http://acervo.ufvjm.edu.br:8080/jspui/handle/1/452

12. Geytenbeek J. Evidence for effective hydrotherapy. Physiotherapy [Internet]. 2002 [cited 2022 Jan 14];88(9):514-29. Available from: https://waterbirth.org/wpcontent/uploads/2017/08/Geytenbeek-Evidence-for-Effective-Hydrotherapy.pdf

13. Eversden L, Maggs F, Nightingale $P$, Jobanputra $P$. A pragmatic randomized controlled trial of hydrotherapy and land exercises on overall well-being and quality of life in rheumatoid arthritis. BMC Musculoskeletal Disorders 2007;8(1):23. doi: 10.1186/14712474-8-23

14. Moreira LDF, Fronza FCAO, Santos RND, Zach PL, Kunii IS, Hayashi LF et al. The benefits of a high-intensity aquatic exercise program (HydrOS) for bone metabolism and bone mass of postmenopausal women. J Bone Miner Metab 2014;32(4):411-19. doi: 10.1007/s00774-013-0509-y

15. Nascimento NAP, Moreira PFP, Marin RV, Moreira LDF, Lazaretti Castro M, Santos CAF et al. Relation among $25(\mathrm{OH}) \mathrm{D}$, aquatic exercises, and multifunctional fitness on functional performance of elderly women from the community. J Nutr Health Aging 2016;20(4):376-82. doi: 10.1007/s12603-015-0569-x

16. Bardin L. Análise de conteúdo, $3^{a}$ reimpressão. São Paulo: Edições 70; 2016. 279p.

17. Sabioni RR, Galante AC, Pelá NTR. The meaning of hydrotherapy: a study with adult women. In: Proceedings of the 8. Brazilian Nursing Communication Symposium; 2002; São Paulo, SP, Brazil.

18. Santana CA, Montenegro SMRS, Costa CSP, Lopes Júnior JEG. Análise da hidroterapia em mulheres com dor lombar e relação com as atividades da vida diária. Fisioter Bras 2014;15(4):263-8. doi: 10.33233/fb.v15i4.352

19. Gomes KV, Zazá DC. Motivos de adesão a prática de atividade física em idosas. Rev Bras Ativ Fís Saúde 2012;14(2):132-38. doi: 10.12820/rbafs.v.14n2p132-138

20. Scliar M. História do conceito de saúde. Physis [Internet] 2007 [cited 2022 Jan 14];17(1):29-41. https://www.scielo.br/j/physis/a/WNtwLvWQRFbscbzCywV9wGq/?format=pdf\&lang=pt

21. Leitão MB, Lazzoli JK, Oliveira MPB, Nóbrega ACL, Silveira GG, Carvalho T, et al. Posicionamento oficial da Sociedade Brasileira de Medicina do Esporte: atividade física e saúde na mulher. Rev Bras Med Esporte [Internet]. 2000[cited 2022 Jan 14];6:215-20. Available from: https://www.medicinadoesporte.org.br/posicionamentos/

22. Viana MF, Almeida $P$, Santos RC. Adaptação portuguesa da versão reduzida do Perfil de Estados de Humor - POMS. Análise Psicológica 2012;19(1):77-92. doi: 10.14417/ap.345

23. Werneck FZ, Navarro CA. Efeito agudo do tipo e da intensidade do exercício sobre os estados de humor. Rev Bras Ativ Fís Saúde 2012;15(4):211-17. doi: 10.12820/rbafs.v.15n4p211-217 
24. Vieira JLL, Da Rocha PGM, Porcu M. Influência do exercício físico no humor e na depressão clínica em mulheres. Motriz Rev Educ Fís [Internet] 2008 [cited 2022 Jan 14];14(2):179-86. Available from: http://bases.bireme.br/cgibin/wxislind.exe/iah/online/?lsisScript=iah/iah.xis\&src=google\&base=LILACS\&lang=p\&n extAction=Ink\&exprSearch $=504278$ \&indexSearch $=$ ID

25. Belza B, Topolski T, Kinne S, Patrick DL, Ramsey SD. Does adherence make a difference? Results from a community-based aquatic exercise program. Nursing Res 2002;51(5):285-91. doi: 10.1097/00006199-200209000-00003 